\title{
Breast-conserving surgery in giant juvenile fibroadenoma: a case report
}

\author{
Angel Millan Juarez ${ }^{1}$, Carla America Suarez Juarez ${ }^{1}$, Ithamar Milagros Arroyo Martinez ${ }^{2}$, \\ Elizabeth Rendon Mondragon ${ }^{3}$
}

${ }^{1}$ Oncology Gynecology Unit, Military Hospital for Women's Specialties and Neonatology, Mexico City, Mexico; ${ }^{2}$ Radiology Unit, Military Hospital for Women's Specialties and Neonatology, Mexico City, Mexico; ${ }^{3}$ Pathology Unit, Military Hospital for Women's Specialties and Neonatology, Mexico City, Mexico

Correspondence to: Angel Millan Juarez. Av. Ings. Militares 29, Lomas Hermosa, Miguel Hidalgo, 11200, Cd. Mx., Mexico. Email: millanjuarezangel33@gmail.com; Carla America Suarez Juarez. Tlacotalpan 59 C400, Roma Sur, Cuauhtemoc, 06760, Cd Mx, Mexico. Email: dra.america.suarez@gmail.com; Ithamar Milagros Arroyo Martinez. Calzada Mexico Tacuba, 1501, Argentina Poniente, Miguel Hidalgo, 11230 , Cd. Mx., Mexico. Email: ithamar_emm@hotmail.com; Elizabeth Rendon Mondragon. Avenida de los Ailes 34, Jardines de San Mateo, Naucalpan, 53240, State of Mexico, 53240, Mexico. Email: beth_ren@hotmail.com.

\begin{abstract}
Giant juvenile fibroadenoma (GJF) represents $0.5 \%$ of fibroadenomas and the usual age range of occurrence is 10 to 18 . GJF definition describes a benign tumor that measures more than $5 \mathrm{~cm}$, weighs more than 500 grams or occupies more than $80 \%$ of the mammary gland. In this report, we present the case of a 14-year-old patient's GJF with six months of evolution. The examination proved skin and the NippleAreola Complex (NAC) involve, resulting in the identification of a smooth, firm and mobile tumor of $20 \mathrm{~cm}$ with negative local lymph nodes. The diagnosis used Mammography (MG), Ultrasound (US), and Magnetic Resonance Imaging (MRI). In addition, Breast Conservative Surgery (BCS) required making an incision in the breast groove, resulting in good aesthetic results. To the best of our knowledge, there is no standard surgical approach. In practice, the treatment options for BCS are enucleation, Wide Local Excision (WLE) and oncoplastic techniques, and Simple Mastectomy (SM) considering or excluding reconstruction. This case challenged medicine itself since human skin gained elasticity after six months, it did not reach full breast development, and hormonal action exposition was present all time, which can generate recurrences, for instance, pregnancy or psychological factors. Whilst GJF is a pathology that requires Therapeutic Board (TB) criteria for adequate BCS, it needs a multidisciplinary team including a Gynecology Oncologist specialist, Plastic and Reconstructive Surgery (PRS) specialist, and a psychologist, where the aesthetic result of the patient should always be priority. This case shows the importance of making adequate differential diagnosis (DD) and respecting oncological principles. In a year of follow-up, skin retraction improved due to normal breast development suggesting effectiveness of the technique.
\end{abstract}

Keywords: Breast; surgery; giant juvenile fibroadenoma; case report

Received: 07 March 2021; Accepted: 20 May 2021; Published: 30 September 2021.

doi: 10.21037/asj-21-10

View this article at: http://dx.doi.org/10.21037/asj-21-10

\section{Introduction}

Breast cancer (BC) is the most common invasive cancer in women. Most times it appears at younger ages. The global incidence of $\mathrm{BC}$ in women aged 10 to 19 according to GLOBOCAN 2020 is 697, with a mortality rate of 192; in
Mexico data is 10 and 2 respectively. Patients' awareness of the disease is increasing; however, it is still very common to treat patients with large tumors in Mexico. GJF is one of many differential diagnoses (DD) of BC (1).

The GJF is a rare and benign breast tumor, representing only $0.5 \%$ of all fibroadenomas. It occurs between 10 to 18 

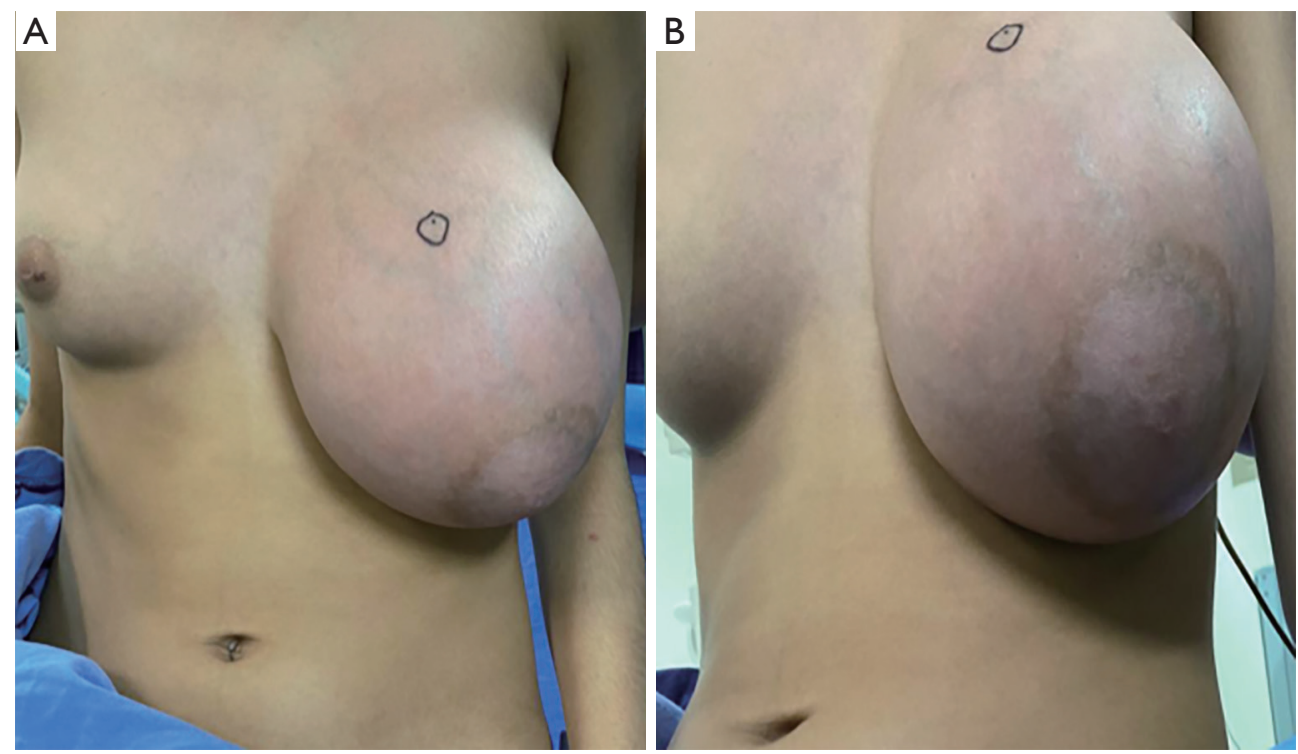

Figure 1 Pre-operative view. (A) Front view. (B) Side view.

years. By definition, this tumor measures more than $5 \mathrm{~cm}$, weighs more than 500 grams or affects more than $80 \%$ of the mammary gland. The clinical presentation is usually very aggressive, with a rapid growth of the tumor and reaching large dimensions, which causes deformity of NippleAreola Complex (NAC), skin, vessels, and mammary gland. Tumors are usually unilateral, although there are reports of bilateralism, and reaching dimensions of more than $20 \mathrm{~cm}$ in exceptional cases. Physical examination usually detects a large, smooth, firm and mobile nodule without associated locoregional lymphadenopathy. The diagnosis requires imaging methods, including Mammography (MG), Ultrasound (US) and Magnetic Resonance Imaging (MRI). A tru-cut biopsy is also necessary to have a histopathological diagnosis. The tumors show benign stromal and epithelial patterns subdivided in two types, pericanalicular and intracanalicular. The differential diagnosis (DD) is good with Phyllodes tumors, lipomas and hamartomas. Malignant transformation is less than $0.3 \%$, and is more common when the GJF is associated with sclerosing adenosis, apocrine papillary changes, calcifications or cysts (2-7). We present the following article in accordance with the CARE reporting checklist (available at https://asj.amegroups.com/ article/view/10.21037/asj-21-10/rc).

\section{Case presentation}

This is a 14-year-old patient with an increase in size of the left breast with 6 months of evolution, accompanied by yellowish secretion from the nipple. Physical examination revealed left breast with increased volume, hyperemic, hyper-vascularized, thin and soft skin, with anatomical loss of the NAC, without secretion, smooth, firm, mobile tumor of dimensions $20 \times 20 \mathrm{~cm}$, and negative locoregional lymph nodes (Figure 1).

MG and US were performed with a BI-RADS 2 report, based on tissue that reflects a cystic pattern of the left breast compatible with Phyllodes Tumor (Figure 2).

MRI with BI-RADS 4A report per nodule showed that it occupied almost the entire left breast, oval, circumscribed, hypodense, with areas of liquid density inside, with axes greater than $16 \times 13 \mathrm{~cm}$, preserved its interface with the pectoral muscle without evidence of infiltration, and fibrograndular tissue displaced in a superior external direction, compatible with GJF (Figure 3).

The case presented to the TB strongly suggested to perform SM of the left breast, instead of BCC, however the latter option prevailed because a benign lesion existed. As MRI has a high negative predictive value in benign lesions, it was decided not to take a biopsy, to avoid a hematoma that could affect the surgical approach or its results.

By having a complete study protocol and normal preoperative studies, the patient treatment involved BCS. The procedure included an incision in the breast groove of approximately $10 \mathrm{~cm}$, exposing the tumor with Allis tweezers and Richardson retractors, separating the entire 

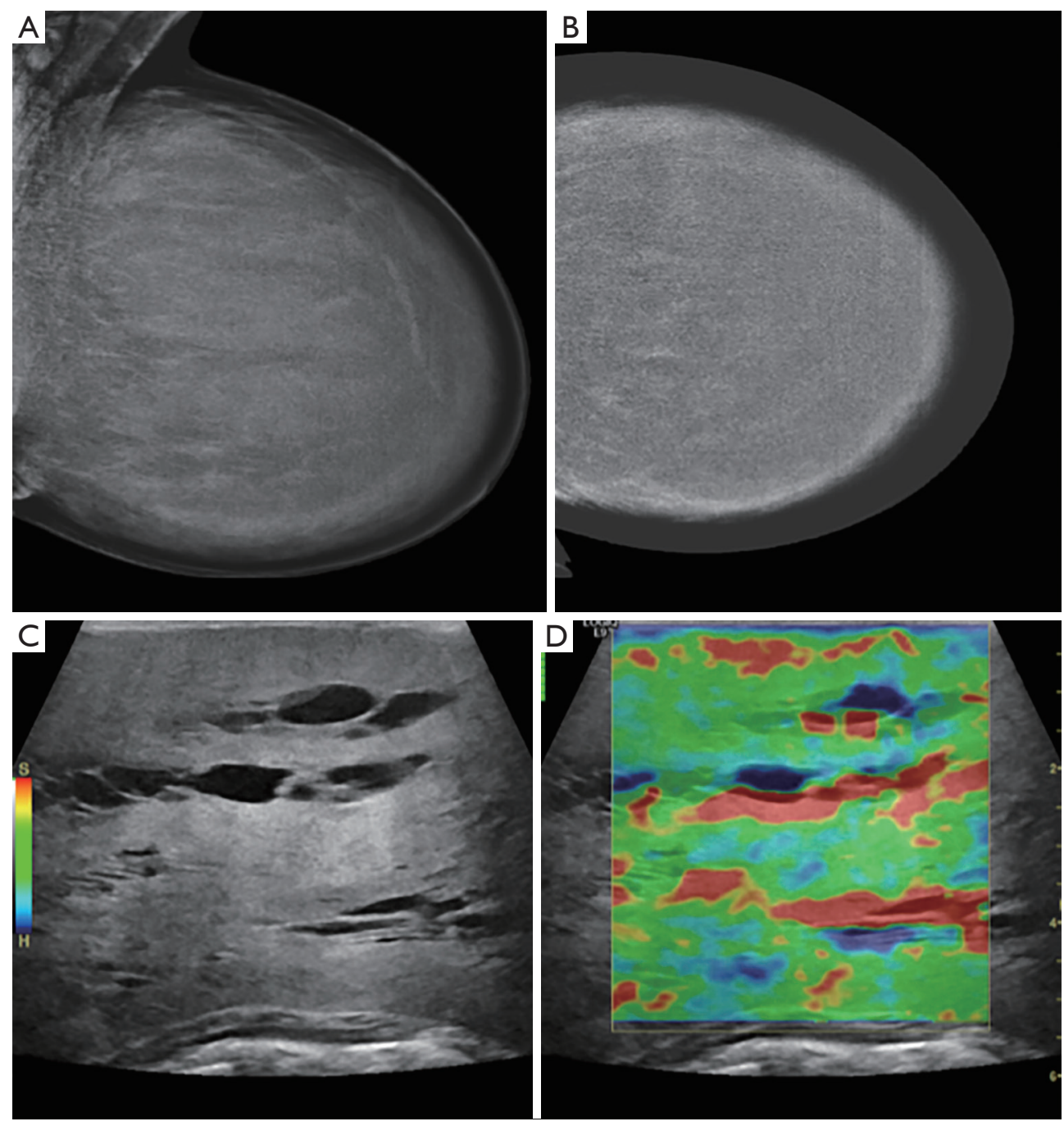

Figure 2 Mammography. (A) Full-field digital mammography in oblique mid-lateral projection of the left breast and (B) tomosynthesis in cephalocaudal projection of the left breast; in which a nodule with intermediate density, oval, circumscribed that occupies the entire mammary gland is identified. (C) Linear gray-scale ultrasound showing the echostructure of the left nodule, which is solid, heterogeneous, with multiple cystic areas inside, some coalescing with each other. (D) Linear ultrasound with application of elastography, where the colorimetric analysis shows intermediate hardness of the left breast nodule.

fascia of the pectoralis major muscle, dissecting the tumor, and cutting the lower pedicle by electrocautery until total extraction. The visualization of the mammary cavity made clear hemostasis. Finally, the incision closed using subdermal 3-0 monocryl stiches required placing a suction drain (Figure 4).

The definitive pathology report described a completely excised giant fibroadenoma with lymphatic vessel ectasia, stromal edema, and long-axis hyaline fibrosis (Figure 5).

The patient secretion lasted four days after surgery and got her drain removed on the sixth day. BCS resulted in a very good aesthetic result for the patient (Figure 6). All procedures performed in the study were in accordance with the ethical standards of the institutional and/or national research committee(s) and with the Helsinki Declaration (as revised in 2013). Written informed consent was obtained from the patient and her legal guradian for publication of this case report and accompanying images. A copy of the written consent is available for review by the editorial office of this journal. 

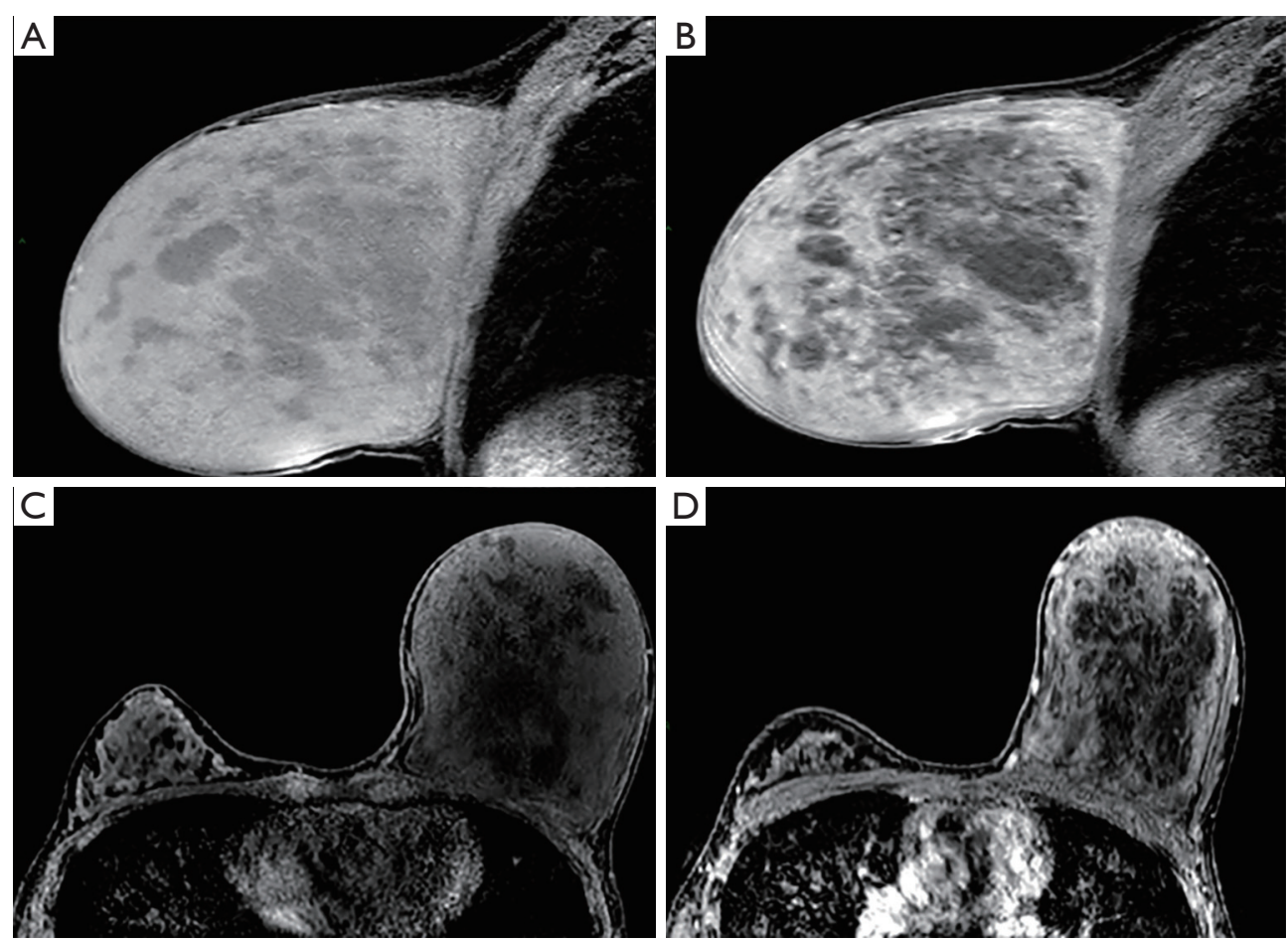

Figure 3 Breast MRI. (A) Sagittal T1 sequence with fat saturation: large nodule that occupies the entire left breast, heterogeneous, with a solid component (hyperintense) and a cystic component (hypointense) in its interior. (B) Sagittal T1 sequence with fat saturation + Gadolinium: intense enhancement of the solid component of the left breast nodule (hyperintense) is observed, the cystic component does not show enhancement, it remains unchanged with intravenous contrast. (C) Axial T1 sequence with fat saturation. (D) Axial T1 sequence with fat saturation + Gadolinium: significant breast asymmetry caused by a breast nodule occupying the entire left mammary gland and producing secondary deformity due to a significant volume effect.

\section{Discussion}

This case shows that the surgical application of the BCS in the GJF is feasible and a mastectomy it is not always necessary as the general practice suggests. This is the novel of our report and our contribution to the medicine literature. The following lines describe the best practice dealing with BCS.

There is no standard surgical approach for GJF, but there are many successful BCS case reports. The treatment options are enucleation, WLE, oncoplastic techniques or SM with or without immediate or delayed Breast Reconstruction Surgery (BRS) (it should be considered that there is a gain of elasticity in the skin in the 6 postoperative months). The type of incision also varies from peri-aerolar, breast groove or axillary incision good cosmetic results are expected when the size ratio of the breast tumor is up to $70 \%(8,9)$.

In our case, to evaluate whether BCS was feasible, we used MRI to calculate the percentage of breast tissue that would remain after the resection of the GJF, it was estimated at $20 \%$. We believe that MRI could be a useful tool for similar cases.

Mammotome is used to reduce $3 \mathrm{~cm}$ of the tumor size and then make a smaller incision in the breast. Tumor resection with Wise pattern skin reduction followed by immediate breast reconstruction with a dermal flap bag is also a treatment option $(10,11)$.

Endovascular embolization of the internal mammary arteries and the lateral thoracic arteries supplying the tumors have been performed before surgery to reduce their vascularity and the risk of bleeding (12).

A challenge in these patients is that they have not yet reached full Tanner $\mathrm{V}$ breast development, which develops between 14.0 to 14.5 years. Therefore, even if we perform onco-plastic techniques or BRS, our effort may require a subsequent re-intervention to re-symmetrize the breasts.

During puberty or pregnancy, the lobes and stroma of 

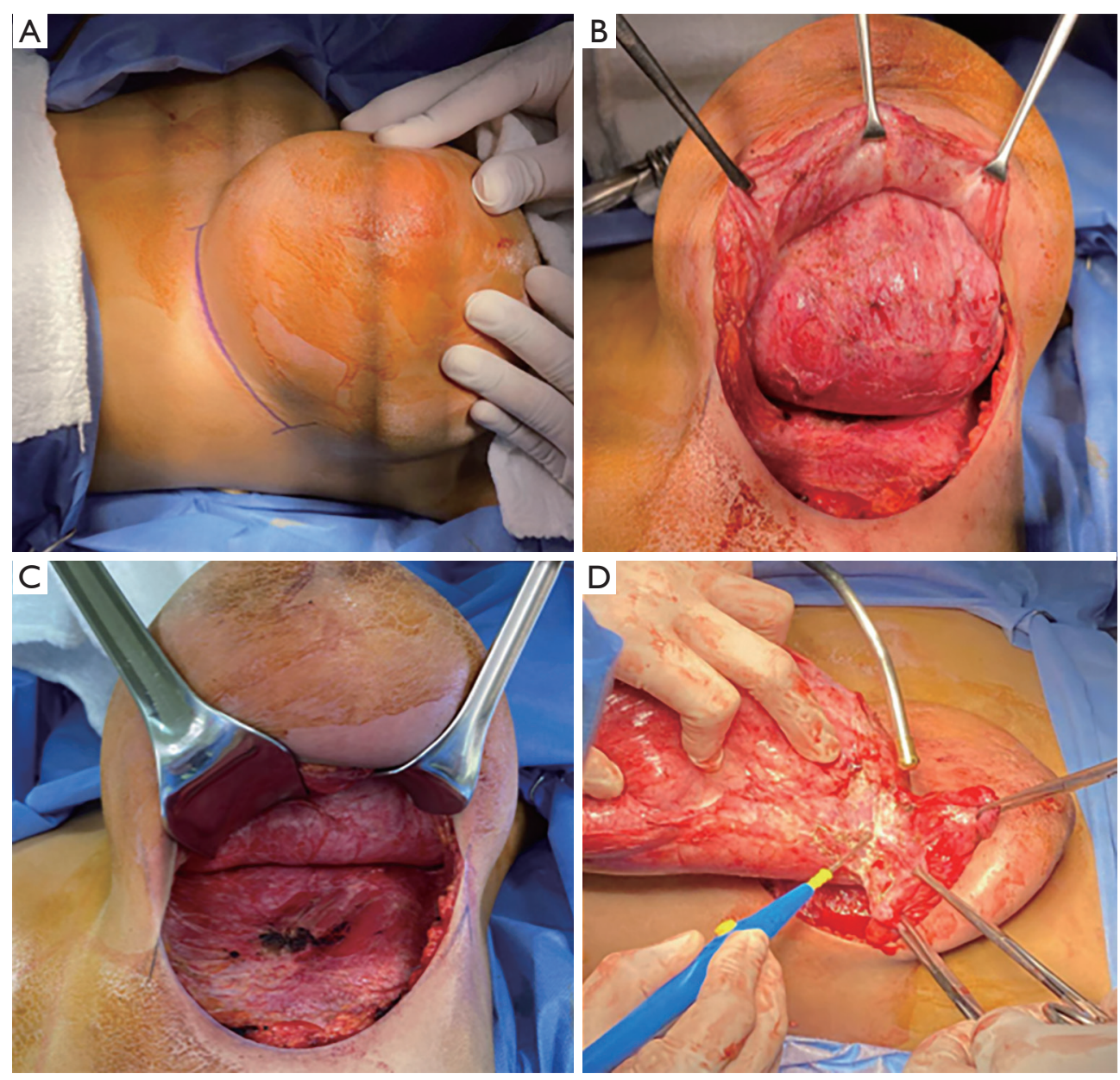

Figure 4 Surgical procedure. (A) Incision in breast groove of approx. $10 \mathrm{~cm}$. (B) Exposure of the tumor with Allis tweezers. (C) Exposure of the tumor. (D) Dissection of the tumor pedicle.

the breast can respond to increased levels of estradiol and progesterone, resulting in an increase in breast size of up to $15 \%$ and $50 \%$ respectively; this condition increases the possibility of recurrence in these patients $(13,14)$.

The main objective is to find the best aesthetic results, since a simple scar on the chest in these adolescent patients may have a strong psychological impact. There are inclusive questionnaires to assess the psychosocial and physical aspects of patients using scores such as the BREAST-Q $(9,11)$.

In this case, our specific objective with this patient was only surveillance without any extra surgical intervention, since it was expected that upon reaching the age 18-20, an aesthetic response close to $100 \%$ would be achieved due to hormonal effect. In a year of follow-up, skin retraction improved due to normal breast development.
PRS has three basic principles: "Preserve all normal mammary parenchyma, adjust the skin and position the NAC symmetrically to the opposite breast." We believe these principles are the cornerstones of BCS which practitioners should consider always (7).

Cautious follow-up is necessary in these patients after treatment, since there are reports that they have predisposition to a higher risk of developing $\mathrm{BC}$ than the general population (15).

\section{Conclusions}

GJF is a pathology that requires broad criteria for conservative surgical management to be adequate and effective, it requires a multidisciplinary treatment that includes the services of Oncological Gynecology, RPS and 

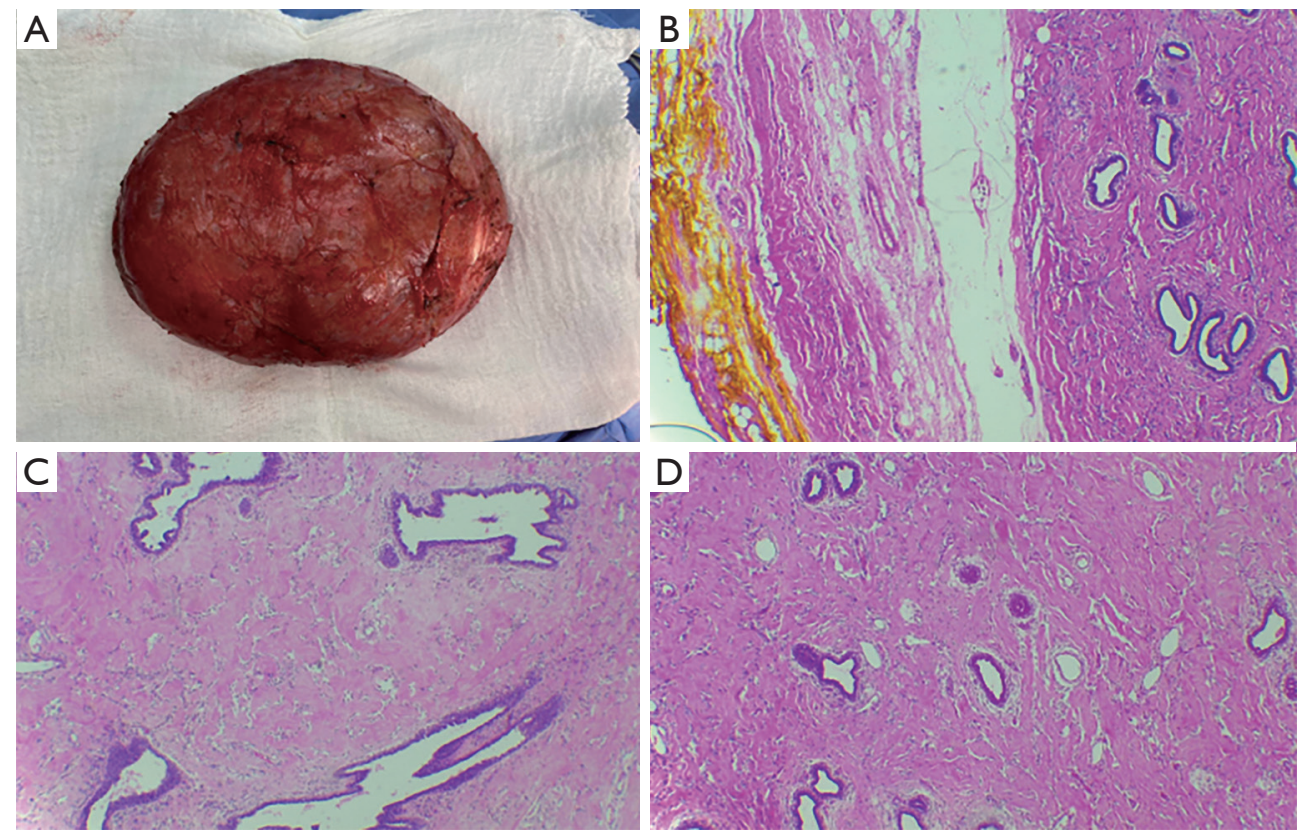

Figure 5 Piece. (A) Macroscopic view. (B) Fibroadenoma showing a thin, yellow-inked fibroconnective capsule (4×). (C,D) Microscopic appearance of fibroadenoma. The glandular epithelium with usual epithelial hyperplasia and the stroma is made of loose connective tissue and partially composed of fibrous tissue $(4 \times)$. Hematoxylin-eosin staining.
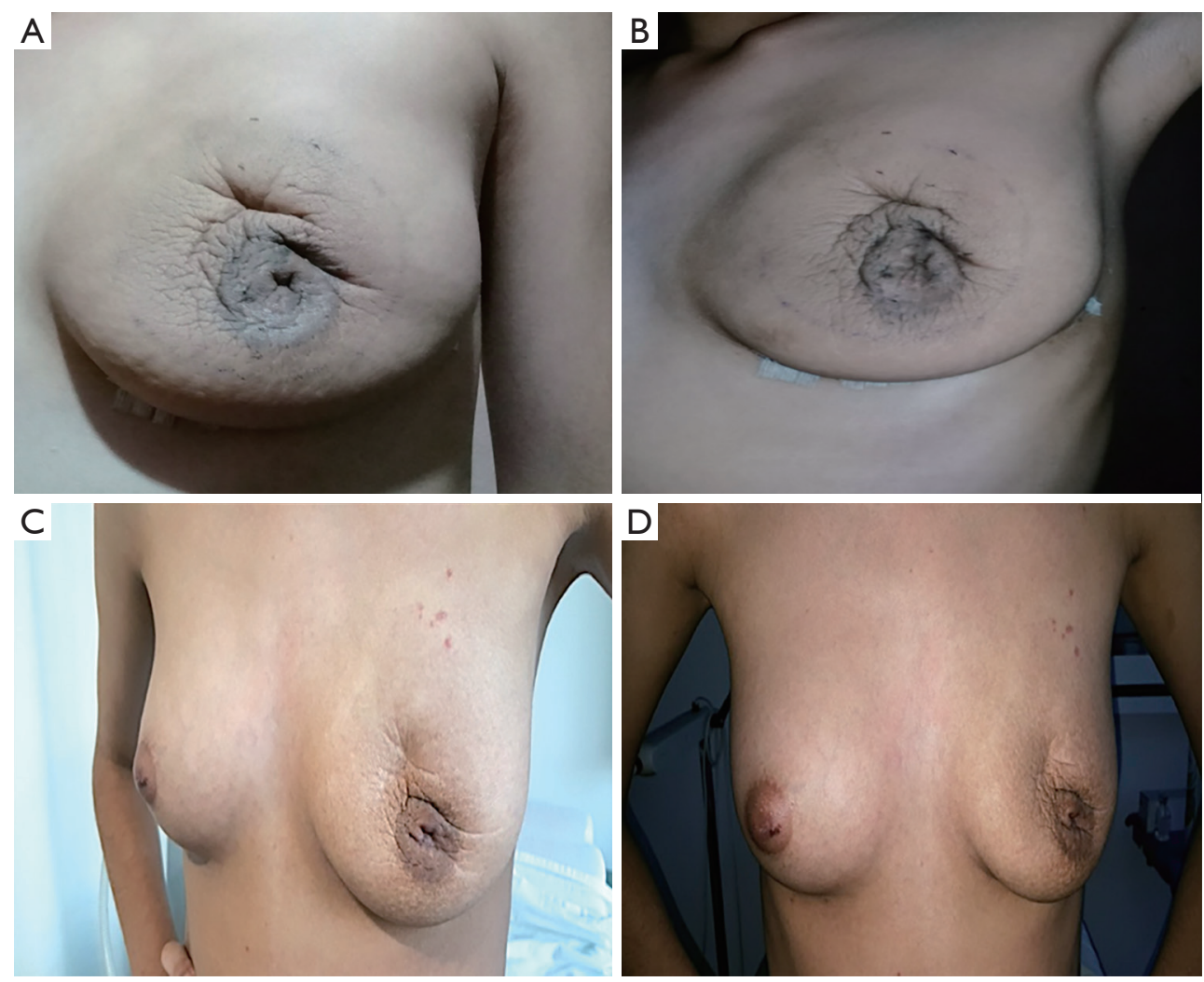

Figure 6 Aesthetic result of the patient. (A,B) Post-operative review at 6 days. (C,D) Post-operative review at 12 months post-treatment. 
psychology, making joint decisions in a JT. The aesthetic outcome of the patient should always be considered to avoid long-term psychological repercussions. Always making an adequate $\mathrm{DD}$, respecting oncological principles and keeping a close follow-up.

\section{Acknowledgments}

The revision of the English translation was carried out by Julian Rodrigo Suarez Juarez from England.

Funding: None.

\section{Footnote}

Reporting Checklist: The authors have completed the CARE reporting checklist. Available at https://asj.amegroups.com/ article/view/10.21037/asj-21-10/rc

Conflicts of Interest: All authors have completed the ICMJE uniform disclosure form (available at https://asj.amegroups. com/article/view/10.21037/asj-21-10/coif). The authors have no conflicts of interest to declare.

Ethical Statement: The authors are accountable for all aspects of the work in ensuring that questions related to the accuracy or integrity of any part of the work are appropriately investigated and resolved. All procedures performed in the study were in accordance with the ethical standards of the institutional and/or national research committee(s) and with the Helsinki Declaration (as revised in 2013). Written informed consent was obtained from the patient and her legal guardian for publication of this case report and accompanying images. A copy of the written consent is available for review by the editorial office of this journal.

Open Access Statement: This is an Open Access article distributed in accordance with the Creative Commons Attribution-NonCommercial-NoDerivs 4.0 International License (CC BY-NC-ND 4.0), which permits the noncommercial replication and distribution of the article with the strict proviso that no changes or edits are made and the original work is properly cited (including links to both the formal publication through the relevant DOI and the license). See: https://creativecommons.org/licenses/by-nc-nd/4.0/.

\section{References}

1. Incidence and mortality of breast cancer in the World and
Mexico in 2020. Available online: https://gco.iarc.fr/today/ online-analysis-table? $\mathrm{v}=2020 \&$ mode $=$ cancer $\&$ mode_popu lation $=$ continents $\&$ population $=900 \&$ populations $=900 \& \mathrm{ke}$ $\mathrm{y}=$ asr \&sex $=0 \&$ cancer $=39 \&$ type $=0 \&$ statistic $=5 \&$ prevalence $=0 \&$ population_group $=0 \&$ ages_group $\% 5 \mathrm{~B} \% 5 \mathrm{D}=3 \&$ ages group $\% 5 \mathrm{~B} \% 5 \mathrm{D}=3 \&$ group_cancer=1\&include_ nmsc $=1 \&$ include_nmsc_other $=1$

2. Giannos A, Stavrou S, Gkali C, et al. A prepubertal giant juvenile fibroadenoma in a 12-year-old girl: Case report and brief literature review. Int J Surg Case Rep 2017;41:427-30.

3. Mohd Firdaus CA, Norjazliney AJ, Abdul Rashid NF. A case report of juvenile giant fibroadenoma of the breast: How common? Ci Ji Yi Xue Za Zhi 2017;29:177-9.

4. Islam S, Saroop $S$, Bheem V, et al. Largest giant juvenile fibroadenoma of the breast. BMJ Case Rep 2019;12:e227277.

5. Jategaonkar PA, Jetegaonkar SP, Yadav SP. Super-Giant Juvenile Breast Fibroadenoma: World's First Case. J Coll Physicians Surg Pak 2018;28:257-8.

6. Makkar N, Singh S, Paul S, et al. Bilateral Giant Juvenile Fibroadenoma of Breast. J Clin Diagn Res 2017;11:ED10-2.

7. Celik SU, Besli Celik D, Yetiskin E, et al. Giant juvenil fibroadenoma of the breast: a clinical case. Arch Argent Pediatr 2017;115:e428-31.

8. Baral S, Gyawali M, Thapa N, et al. Giant juvenile fibroadenoma in an adolescent female: A case report. Clin Case Rep 2020;8:3489-93.

9. Lim GH, Ng RP, Leong LCH. Development of a surgical algorithm by using pre-operative imaging to predict mammoplasty cosmetic outcomes for large non-malignant tumours. Gland Surg 2017;6:649-53.

10. Wang T, Zhu L. Mammotome-assisted removal with minimal incision of large juvenile fibroadenoma of breast: A case report. Medicine (Baltimore) 2020;99:e19442.

11. Du F, Dong R, Zeng A, et al. Surgical management of giant juvenile fibroadenoma with skin reducing tumor resection and immediate breast reconstruction: A singlecenter experience. J Surg Oncol 2020;121:441-6.

12. Rafeek N, Rangasami R, Dhanraj K, et al. Multimodality approach in the diagnosis and management of bilateral giant juvenile breast fibroadenoma. BMJ Case Rep 2016;2016:bcr2016217588.

13. Huang IC, Li PC, Ding DC. Recurrent juvenile fibroadenoma of the breast in an adolescent: a case report. Medicine 2018;97:e10765

14. Moghimi M, Soltani HR, Zarch MB. Giant Juvenile 
Fibroadenoma Presenting in a 15-Year-Old Pregnant Woman: A Case Report. J Ped Hematol Oncol 2017;7:130-2.

doi: $10.21037 /$ asj-21-10

Cite this article as: Juarez AM, Juarez CAS, Martinez IMA, Mondragon ER. Breast-conserving surgery in giant juvenile fibroadenoma: a case report. AME Surg J 2021;1:18.
15. El-Wakeel H, Umpleby HC. Systematic review of fibroadenoma as a risk factor for breast cancer. Breast 2003;12:302-7. 